\title{
Left (Right)-Quasi Neutrosophic Triplet Loops (Groups) and Generalized BE-Algebras
}

\author{
Xiaohong Zhang ${ }^{1,2, *(\mathbb{D})}$, Xiaoying $\mathrm{Wu}^{1}{ }^{1}$, Florentin Smarandache ${ }^{3}\left[{ }^{(0)}\right.$ and Minghao $\mathrm{Hu}{ }^{1}$ \\ 1 Department of Mathematics, Shaanxi University of Science \& Technology, Xi'an 710021, China; \\ 46018@sust.edu.cn(X.W.); huminghao@sust.edu.cn (M.H.) \\ 2 Department of Mathematics, Shanghai Maritime University, Shanghai 201306, China \\ 3 Department of Mathematics, University of New Mexico, Gallup, NM 87301, USA; smarand@unm.edu \\ * Correspondence: zhangxiaohong@sust.edu.cn or zhangxh@shmtu.edu.cn; Tel.: +86-029-8616-8320
}

Received: 29 May 2018; Accepted: 19 June 2018; Published: 26 June 2018

\begin{abstract}
The new notion of a neutrosophic triplet group (NTG) is proposed by Florentin Smarandache; it is a new algebraic structure different from the classical group. The aim of this paper is to further expand this new concept and to study its application in related logic algebra systems. Some new notions of left (right)-quasi neutrosophic triplet loops and left (right)-quasi neutrosophic triplet groups are introduced, and some properties are presented. As a corollary of these properties, the following important result are proved: for any commutative neutrosophic triplet group, its every element has a unique neutral element. Moreover, some left (right)-quasi neutrosophic triplet structures in BE-algebras and generalized BE-algebras (including CI-algebras and pseudo CI-algebras) are established, and the adjoint semigroups of the BE-algebras and generalized $\mathrm{BE}$-algebras are investigated for the first time.
\end{abstract}

Keywords: neutrosophic triplet; quasi neutrosophic triplet loop; quasi neutrosophic triplet group; BE-algebra; CI-algebra

\section{Introduction}

The symmetry exists in the real world, and group theory is a mathematical tool for describing symmetry. At the same time, in order to describe the generalized symmetry, the concept of group is popularized in different ways, for example, the notion of a generalized group is introduced (see [1-4]). Recently, F. Smarandache [5,6] introduced another new algebraic structure, namely: neutrosophic triplet group, which comes from the theory of the neutrosophic set (see [7-11]). As a new extension of the concept of group, the neutrosophic triplet group has attracted the attention of many scholars, and a series of related papers have been published [12-15].

On the other hand, in the last twenty years, the non-classical logics, such as various fuzzy logics, have made great progress. At the same time, the research on non-classical logic algebras that are related to it have also made great achievements [16-26]. As a generalization of BCK-algebra, H.S. Kim and Y.H. Kim [27] introduced the notion of BE-algebra. Since then, some scholars have studied ideals (filters), congruence relations of BE-algebras, and various special BE-algebras have been proposed, these research results are included in the literature [28-31] and monograph [32]. In 2013 and 2016, the new notions of pseudo BE-algebra and commutative pseudo BE-algebra were introduced, and some new properties were obtained [33,34]. Similar to BCI-algebra as a generalization of BCK-algebra, B.L. Meng introduced the concept of CI-algebra, which is as a generalization of BE-algebra, and studied the structures and closed filters of CI-algebras [35-37]. After that, the CI-algebras and their related algebraic structures (such as Q-algebras, pseudo Q-algebras, pseudo CI-algebras, and pseudo $\mathrm{BCH}$ algebras) have been extensively studied [38-46]. 
This paper will combine the above two directions to study general neutrosophic triplet structures and the relationships between these structures and generalized BE-algebras. On the one hand, we introduce various general neutrosophic triplet structures, such as $(l-l)$-type, $(l-r)$-type, $(r-l)$-type, $(r-r)$-type, (l-lr)-type, $(r-l r)$-type, $(l r-l)$-type, and (lr-r)-type quasi neutrosophic triplet loops (groups), and investigate their basic properties. Moreover, we get an important corollary, namely: that for any commutative neutrosophic triplet group, its every element has a unique neutral element. On the other hand, we further study the properties of (pseudo) BE-algebras and (pseudo) CI-algebras, and the general neutrosophic triplet structures that are contained in a BE-algebra (CI-algebra) and pseudo BE-algebra (pseudo CI-algebra). Moreover, for the first time, we introduce the concepts of adjoint semigroups of BE-algebras and generalized BE-algebras (including CI-algebras, pseudo BE-algebras, and pseudo CI-algebras) and discuss some interesting topics.

\section{Basic Concepts}

Definition 1. ([5,6]) Let $N$ be a set together with a binary operation *. Then, $N$ is called a neutrosophic triplet set if, for any $a \in N$, there exists a neutral of ' $a$ ', called neut( $(a)$, and an opposite of ' $a$ ', called anti(a), with neut( $(a)$ and anti(a), belonging to $N$, such that:

$$
\begin{gathered}
a * \operatorname{neut}(a)=\operatorname{neut}(a) * a=a ; \\
a * \operatorname{anti}(a)=\operatorname{anti}(a) * a=\operatorname{neut}(a) .
\end{gathered}
$$

It should be noted that neut(a) and anti(a) may not be unique here for some $a \in N$. We call $(a$, neut $(a)$, and $\operatorname{anti}(a))$ a neutrosophic triplet for the determined neut $(a)$ and anti(a).

Remark 1. In the original definition, the neutral element is different from the unit element in the traditional group theory. The above definition of this paper takes away such restriction, please see the Remark 3 in Ref. [12].

Definition 2. $([5,6,13])$ Let $\left(N,{ }^{*}\right)$ be a neutrosophic triplet set.

(1) If ${ }^{*}$ is well-defined, that is, for any $a, b \in N$, one has $a * b \in N$. Then, $N$ is called a neutrosophic triplet loop.

(2) If $N$ is a neutrosophic triplet loop, and ${ }^{*}$ is associative, that is, $\left(a^{*} b\right)^{*} c=a^{*}\left(b^{*} c\right)$ for all $a, b, c \in N$. Then, $N$ is called a neutrosophic triplet group.

(3) If $N$ is a neutrosophic triplet group, and * is commutative, that is, $a * b=b^{*}$ a for all $a, b \in N$. Then, $N$ is called a commutative neutrosophic triplet group.

Definition 3. ([27,35,41,42]) A CI-algebra (dual Q-algebra) is an algebra $(X ; \rightarrow, 1)$ of type $(2,0)$, satisfying the following conditions:

(i) $x \rightarrow x=1$,

(ii) $1 \rightarrow x=x$,

(iii) $x \rightarrow(y \rightarrow z)=y \rightarrow(x \rightarrow z)$, for all $x, y, z \in X$.

A CI-algebra $(X ; \rightarrow, 1)$ is called a BE-algebra, if it satisfies the following axiom:

(iv) $x \rightarrow 1=1$, for all $x \in X$.

A CI-algebra $(\mathrm{X} ; \rightarrow, 1)$ is called a dual BCH-algebra, if it satisfies the following axiom:

(v) $x \rightarrow y=y \rightarrow x=1 \Rightarrow x=y$.

A binary relation $\leq$ on CI-algebra (BE-algebra) $X$, is defined by $x \leq y$ if, and only if, $x \rightarrow y=1$. 
Definition 4. ([33,43,45]) An algebra $(X ; \rightarrow, \rightsquigarrow, 1)$ of type $(2,2,0)$ is called a dual pseudo Q-algebra if, for all $x, y, z \in X$, it satisfies the following axioms:

$($ dpsQ1) $x \rightarrow x=x \rightsquigarrow x=1$,

$($ dpsQ2) $1 \rightarrow x=1 \rightsquigarrow x=x$,

(dpsQ3) $x \rightarrow(y \rightsquigarrow z)=y \rightsquigarrow(x \rightarrow z)$.

A dual pseudo Q-algebra X is called a pseudo CI-algebra, if it satisfies the following condition:

(psCI) $x \rightarrow y=1 \Leftrightarrow x \rightsquigarrow y=1$.

A pseudo CI-algebra $X$ is called a pseudo BE-algebra, if it satisfies the following condition:

(psBE) $x \rightarrow 1=x \rightsquigarrow 1=1$, for all $x \in X$.

A pseudo CI-algebra $X$ is called a pseudo BCH-algebra, if it satisfies the following condition:

(psBCH) $x \rightarrow y=y \rightsquigarrow x=1 \Rightarrow x=y$.

In a dual pseudo-Q algebra, one can define the following binary relations:

$$
x \leq \rightarrow y \Leftrightarrow x \rightarrow y=1 . x \leq \rightsquigarrow y \Leftrightarrow x \rightsquigarrow y=1 .
$$

Obviously, a dual pseudo-Q algebra $X$ is a pseudo CI-algebra if, and only if, $\leq_{\rightarrow}=\leq_{m}$.

\section{Various Quasi Neutrosophic Triplet Loops (Groups)}

Definition 5. Let $N$ be a set together with a binary operation * (that is, $\left(N,{ }^{*}\right)$ be a loop) and $a \in N$.

(1) If exist $b, c \in N$, such that $a * b=a$ and $a{ }^{*} c=b$, then a is called an NT-element with ( $r$-r)-property;

(2) If exist $b, c \in N$, such that $a^{*} b=a$ and $c^{*} a=b$, then $a$ is called an NT-element with ( $r$-l)-property;

(3) If exist $b, c \in N$, such that $b^{*} a=a$ and $c^{*} a=b$, then $a$ is called an NT-element with (l-l)-property;

(4) If exist $b, c \in N$, such that $b^{*} a=a$ and $a{ }^{*} c=b$, then $a$ is called an NT-element with (l-r)-property;

(5) If exist $b, c \in N$, such that $a^{*} b=b^{*} a=a$ and $c^{*} a=b$, then $a$ is called an NT-element with (lr-l)-property;

(6) If exist $b, c \in N$, such that $a^{*} b=b^{*} a=a$ and $a{ }^{*} c=b$, then $a$ is called an NT-element with (lr-r)-property;

(7) If exist $b, c \in N$, such that $b^{*} a=a$ and $a{ }^{*} c=c^{*} a=b$, then $a$ is called an NT-element with (l-lr)-property;

(8) If exist $b, c \in N$, such that $a^{*} b=a$ and $a^{*} c=c{ }^{*} a=b$, then $a$ is called an NT-element with (r-lr)-property;

(9) If exist $b, c \in N$, such that $a * b=b^{*} a=a$ and $a{ }^{*} c=c^{*} a=b$, then $a$ is called an NT-element with (lr-lr)-property.

It is easy to verify that, (i) if $a$ is an NT-element with (l-lr)-property, then $a$ is an NT-element with (l-l)-property and (l-r)-property; if $a$ is an NT-element with (lr-l)-property, then $a$ is an NT-element with $(l-l)$-property and $(r-l)$-property; and so on; (ii) a neutrosophic triplet loop $\left(N,{ }^{*}\right)$ is a neutrosophic triplet group if, and only if, every element in $N$ is an NT-element with (lr-lr)-property; (iii) if * is commutative, then the above properties coincide. Moreover, the following example shows that $(r-l)$-property and $(r-r)$-property cannot infer to $(r-l r)$-property, and $(r-r)$-property and $(l-l r)$-property cannot infer to $(l r-l r)$-property.

Example 1. Let $N=\{a, b, c, d\}$. The operation * on $N$ is defined as Table 1. Then, $\left(N,{ }^{*}\right)$ is a loop, and $a$ is an NT-element with (lr-lr)-property; $b$ is an NT-element with (lr-r)-property; $c$ is an NT-element with (r-l)-property and $(r-r)$-property, but $c$ is not an NT-element with ( $r$-lr)-property; and $d$ is an NT-element with ( $r$-r)-property and (l-lr)-property, but $d$ is not an NT-element with (lr-lr)-property. 
Table 1. Neutrosophic triplet (NT)-elements in a loop.

\begin{tabular}{lllll}
\hline$*$ & $\boldsymbol{a}$ & $\boldsymbol{b}$ & $\boldsymbol{c}$ & $\boldsymbol{d}$ \\
\hline $\boldsymbol{a}$ & $a$ & $a$ & $a$ & $d$ \\
$\boldsymbol{b}$ & $c$ & $a$ & $b$ & $c$ \\
$c$ & $c$ & $b$ & $d$ & $a$ \\
$d$ & $a$ & $d$ & $b$ & $a$ \\
\hline
\end{tabular}

Definition 6. Let $(N, *)$ be a loop (semi-group). If for every element a in $N, a$ is an NT-element with $\left(r\right.$-r)-property, then $\left(N,{ }^{*}\right)$ is called $(r-r)$-quasi neutrosophic triplet loop (group). Similarly, if for every element $a$ in $N, a$ is an NT-element with $(r-l)-,(l-l)-,(l-r)-,(l r-l)-,(l r-r)-,(l-l r)-,(r-l r)$-property, then $\left(N,{ }^{*}\right)$ is called $(r-l)_{-},(l-l)_{-},(l-r)-,(l r-l)_{-},(l r-r)-,(l-l r)_{-},(r-l r)-q u a s i$ neutrosophic triplet loop (group), respectively. All of these generalized neutrosophic triplet loops (groups) are collectively known as quasi neutrosophic triplet loops (groups).

Remark 2. For quasi neutrosophic triplet loops (groups), we will use the notations like neutrosophic triplet loops (groups), for example, to denote a $(r-r)$-neutral of ' $a$ ' by neut ${ }_{(r-r)}(a)$, denote a $(r-r)$-opposite of ' $a$ ' by anti $i_{(r-r)}(a)$, where ' $a$ ' is an NT-element with $(r-r)$-property. If neut $t_{(r-r)}(a)$ and anti $i_{(r-r)}(a)$ are not unique, then denote the set of all (r-r)-neutral of ' $a$ ' by $\left\{\right.$ neut $\left.t_{(r-r)}(a)\right\}$, denote the set of all $(r-r)$-opposite of ' $a$ ' by $\left\{\right.$ anti $\left.i_{(r-r)}(a)\right\}$.

For the loop $\left(N,{ }^{*}\right)$ in Example 1 , we can verify that $\left(N,{ }^{*}\right)$ is a $(r-r)$-quasi neutrosophic triplet loop, and we have the following:

$$
\begin{gathered}
\operatorname{neut}_{(r-r)}(a)=a, \operatorname{anti}_{(r-r)}(a)=a ; \operatorname{neut}_{(r-r)}(b)=c,\left\{\operatorname{anti}_{(r-r)}(b)\right\}=\{a, d\} ; \\
\operatorname{neut}_{(r-r)}(c)=a, \operatorname{anti}_{(r-r)}(c)=d ; \operatorname{neut}_{(r-r)}(d)=b, \operatorname{anti}_{(r-r)}(d)=c .
\end{gathered}
$$

Theorem 1. If $\left(N,{ }^{*}\right)$ is a (l-lr)-quasi neutrosophic triplet group, then $\left(N,{ }^{*}\right)$ is a neutrosophic triplet group. Moreover, if $(N, *)$ is a $\left(r\right.$-lr)-quasi neutrosophic triplet group, then $\left(N,{ }^{*}\right)$ is a neutrosophic triplet group.

Proof. Suppose that $\left(N,{ }^{*}\right)$ is a $(l-l r)$-quasi neutrosophic triplet group. For any $a \in N$, by Definitions 5 and 6, we have the following:

$$
\operatorname{neut}_{(l-l r)}(a) * a=a, \operatorname{anti}_{(l-l r)}(a) * a=a * \operatorname{anti}_{(l-l r)}(a)=\operatorname{neut}_{(l-l r)}(a) .
$$

Here, $\operatorname{neut}_{(l-l r)}(a) \in\left\{\operatorname{neut}_{(l-l r)}(a)\right\}, \operatorname{anti}_{(l-l r)}(a) \in\left\{\operatorname{anti}_{(l-l r)}(a)\right\}$. Applying associative law we get the following:

$$
a * \operatorname{neut}_{(l-l r)}(a)=a *\left(\operatorname{anti}_{(l-l r)}(a) * a\right)=\left(a * \operatorname{anti}_{(l-l r)}(a)\right) * a=\operatorname{neut}_{(l-l r)}(a) * a=a .
$$

This means that neut $(l-l r)(a)$ is a right neutral of ' $a$ '. From the arbitrariness of $a$, it is known that $(N, *)$ is a neutrosophic triplet group.

Another result can be proved similarly.

Theorem 2. Let $(N, *)$ be a $(r$-lr)-quasi neutrosophic triplet group such that:

$$
\left(s^{*} p\right) * a=a^{*}\left(s^{*} p\right), \forall s \in\left\{\operatorname{neut}_{(r-l r)}(a)\right\}, \forall p \in\left\{\operatorname{anti}_{(r-l r)}(a)\right\} .
$$

Then,

(1) for any $a \in N, s \in\left\{\right.$ neut $\left._{(r-l r)}(a)\right\} \Rightarrow s{ }^{*} s=s$.

(2) for any $a \in N, s, t \in\left\{\right.$ neut $\left._{(r-l r)}(a)\right\} \Rightarrow s^{*} t=t$.

(3) when * is commutative, for any $a \in N$, neut $(r-l r)(a)$ is unique. 
Proof. (1) Assume $s \in\left\{\right.$ neut $\left._{(r-l r)}(a)\right\}$, then $a^{*} s=a$, and exist $p \in N$, such that $p^{*} a=a^{*} p=s$. Thus,

$$
\begin{gathered}
\left(s^{*} p\right) * a=s^{*}(p * a)=s^{*} s, \\
a *\left(s^{*} p\right)=\left(a^{*} s\right) * p=a^{*} p=s .
\end{gathered}
$$

According to the hypothesis, $\left(s^{*} p\right) * a=a^{*}\left(s^{*} p\right)$, it follows that $s^{*} s=s$.

(2) Assume $s, t \in\left\{\right.$ neut $\left._{(r-l r)}(a)\right\}$, then $a^{*} s=a, a^{*} t=a$, and exist $p, q \in N$, such that $p^{*} a=a^{*} p=s, q^{*}$ $a=a * q=t$. Thus,

$$
\begin{gathered}
\left(s^{*} q\right) * a=s^{*}\left(q^{*} a\right)=s^{*} t, \\
a^{*}\left(s^{*} q\right)=\left(a^{*} s\right) * q=a * q=t .
\end{gathered}
$$

According to the hypothesis, $\left(s^{*} p\right)^{*} a=a^{*}\left(s^{*} p\right)$, it follows that $s^{*} t=t$.

(3) Suppose $a \in N, s, t \in\left\{\right.$ neut $\left.t_{(r-l)}(a)\right\}$. Applying Theorem (2) to $s$ and $t$ we have $s^{*} t=t$. Moreover, applying Therorem (2) to $t$ and $s$ we have $t^{*} s=s$. Hence, when * is commutative, $s^{*} t=t^{*} s$. Therefore, $s=t$, that is, neut $_{(r-l r)}(a)$ is unique.

Corollary 1. Let $(N, *)$ be a commutative neutrosophic triplet group. Then neut (a) is unique for any a $\in N$.

Proof. Since all neutrosophic triplet groups are $(r-l r)$-quasi neutrosophic triplet groups, and ${ }^{*}$ is commutative, then the assumption conditions in Theorem 2 are valid for $N$, so applying Theorem 2 (3), we get that neut(a) is unique for any $a \in N$.

The following examples show that the neutral element may be not unique in the neutrosophic triplet loop.

Example 2. Let $N=\{1,2,3\}$. Define binary operation * on $N$ as following Table 2 . Then, $(N, *)$ is a commutative neutrosophic triplet loop, and $\{$ neut $(1)\}=\{1,2\}$. Since $(1 * 3) * 3 \neq 1 *(3 * 3)$, so $(N, *)$ is not a neutrosophic triplet group.

Table 2. Commutative neutrosophic triplet loop.

\begin{tabular}{llll}
\hline$*$ & $\mathbf{1}$ & $\mathbf{2}$ & $\mathbf{3}$ \\
\hline $\mathbf{1}$ & 1 & 1 & 2 \\
$\mathbf{2}$ & 1 & 2 & 3 \\
$\mathbf{3}$ & 2 & 3 & 3 \\
\hline
\end{tabular}

Example 3. Let $N=\{1,2,3,4\}$. Define binary operation * on $N$ as following Table 3. Then, $(N, *)$ is a neutrosophic triplet loop, and $\{$ neut $(4)\}=\{2,3\}$. Since $\left(4^{*} 1\right) * 1 \neq 4 *(1 * 1)$, so $(N, *)$ is not a neutrosophic triplet group.

Table 3. Non-commutative neutrosophic triplet loop.

\begin{tabular}{lllll}
\hline$*$ & $\mathbf{1}$ & $\mathbf{2}$ & $\mathbf{3}$ & $\mathbf{4}$ \\
\hline $\mathbf{1}$ & 3 & 1 & 1 & 3 \\
$\mathbf{2}$ & 4 & 2 & 2 & 4 \\
$\mathbf{3}$ & 1 & 3 & 3 & 4 \\
$\mathbf{4}$ & 3 & 4 & 4 & 2 \\
\hline
\end{tabular}




\section{Quasi Neutrosophic Triplet Structures in BE-Algebras and CI-Algebras}

From the definition of BE-algebra and CI-algebra (see Definition 3), we can see that ' 1 ' is a left neutral element of every element, that is, BE-algebras and CI-algebras are directly related to quasi neutrosophic triplet structures. This section will reveal the various internal connections among them.

\subsection{BE-Algebras (CI-Algebras) and (l-l)-Quasi Neutrosophic Triplet Loops}

Theorem 3. Let $(X ; \rightarrow, 1)$ be a BE-algebra. Then $(X, \rightarrow)$ is a (l-l)-quasi neutrosophic triplet loop. And, when $|X|>1,(X, \rightarrow)$ is not a (lr-l)-quasi neutrosophic triplet loop with neutral element 1.

Proof. By Definition 3, for all $x \in X, 1 \rightarrow x=x$ and $x \rightarrow x=1$. According Definition 6, we know that $(X, \rightarrow)$ is a $(l-l)$-quasi neutrosophic triplet loop, such that:

$$
1 \in\left\{\operatorname{neut}_{(l-l)}(x)\right\}, x \in\left\{\operatorname{anti}_{(-l-l)}(x)\right\} \text {, for any } x \in X \text {. }
$$

If $|X|>1$, then exist $x \in X$, such that $x \neq 1$. Using Definition 3 (iv), $x \rightarrow 1=1 \neq x$, this means that 1 is not a right neutral element of $x$. Hence, $(X, \rightarrow)$ is not a $(l r-l)$-quasi neutrosophic triplet loop with neutral element 1 .

Example 4. Let $X=\{a, b, c, 1\}$. Define binary operation * on $N$ as following Table 4 . Then, $(X ; \rightarrow, 1)$ is a $B E$-algebra, and $(X, \rightarrow)$ is a (l-l)-quasi neutrosophic triplet loop, such that:

$$
\begin{gathered}
\left\{\operatorname{neut}_{(l-l)}(a)\right\}=\{1\},\left\{\operatorname{anti}_{(l-l)}(a)\right\}=\{a, c\} ;\left\{\text { neut }_{(l-l)}(b)\right\}=\{1\},\left\{\operatorname{anti}_{(l-l)}(b)\right\}=\{b, c\} ; \\
\left\{\operatorname{neut}_{((-l))}(c)\right\}=\{1\},\left\{\operatorname{anti}_{((-))}(c)\right\}=\{c\} ;\left\{\text { neut }_{(l-l)}(1)\right\}=\{1\},\left\{\operatorname{anti}_{(-l)}(1)\right\}=\{1\} .
\end{gathered}
$$

Table 4. BE-algebra and (l-l)-quasi neutrosophic triplet loop (1).

\begin{tabular}{ccccc}
\hline$\rightarrow$ & $\boldsymbol{a}$ & $\boldsymbol{b}$ & $\boldsymbol{c}$ & $\mathbf{1}$ \\
\hline $\boldsymbol{a}$ & 1 & $b$ & $b$ & 1 \\
$\boldsymbol{b}$ & $a$ & 1 & $a$ & 1 \\
$\boldsymbol{c}$ & 1 & 1 & 1 & 1 \\
$\mathbf{1}$ & $a$ & $b$ & $c$ & 1 \\
\hline
\end{tabular}

Example 5. Let $X=\{a, b, c, 1\}$. Define binary operation * on $N$ as following Table 5 . Then, $(X ; \rightarrow, 1)$ is a $B E$-algebra, and $(X, \rightarrow)$ is a (l-l)-quasi neutrosophic triplet loop such that:

$$
\begin{aligned}
& \left\{\operatorname{neut}_{(l-l)}(a)\right\}=\{1\},\left\{\operatorname{anti}_{(l-l)}(a)\right\}=\{a\} ;\left\{\text { neut }_{(l-l)}(b)\right\}=\{1\},\left\{\operatorname{anti}_{(l-l)}(b)\right\}=\{b\} ; \\
& \left\{\operatorname{neut}_{(l-l)}(c)\right\}=\{1\},\left\{\operatorname{anti}_{((-l))}(c)\right\}=\{c\} ;\left\{\text { neut }_{((-l))}(1)\right\}=\{1\},\left\{\operatorname{anti}_{((-l))}(1)\right\}=\{1\} .
\end{aligned}
$$

Table 5. BE-algebra and (l-l)-quasi neutrosophic triplet loop (2).

\begin{tabular}{ccccc}
\hline$\rightarrow$ & $\boldsymbol{a}$ & $\boldsymbol{b}$ & $c$ & $\mathbf{1}$ \\
\hline $\boldsymbol{a}$ & 1 & $b$ & $c$ & 1 \\
$\boldsymbol{b}$ & $a$ & 1 & $c$ & 1 \\
$c$ & $a$ & $b$ & 1 & 1 \\
$\mathbf{1}$ & $a$ & $b$ & $c$ & 1 \\
\hline
\end{tabular}


Definition 7. ([36]) Let $(X ; \rightarrow, 1)$ be $a$ CI-algebra and $a \in X$. If for any $x \in X, a \rightarrow x=1$ implies $a=x$, then $a$ is called an atom in $X$. Denote $A(X)=\{a \in X \mid a$ is an atom in $X\}$, it is called the singular part of $X$. A CI-algebra $(X ; \rightarrow, 1)$ is said to be singular if every element of $X$ is an atom.

Lemma 1. ([35-37]) If $(X ; \rightarrow, 1)$ is a CI-algebra, then for all $x, y \in X$ :

(1) $x \rightarrow((x \rightarrow y) \rightarrow y)=1$,

(2) $1 \rightarrow x=1$ (or equivalently, $1 \leq x$ ) implies $x=1$,

(3) $(x \rightarrow y) \rightarrow 1=(x \rightarrow 1) \rightarrow(y \rightarrow 1)$.

Lemma 2. ([36]) Let $(X ; \rightarrow, 1)$ be a CI-algebra. If $a, b \in X$ are atoms in $X$, then the following are true:

(1) $a=(a \rightarrow 1) \rightarrow 1$,

(2) $(a \rightarrow b) \rightarrow 1=b \rightarrow a$,

(3) $((a \rightarrow b) \rightarrow 1) \rightarrow 1=a \rightarrow b$,

(4) for any $x \in X,(a \rightarrow x) \rightarrow(b \rightarrow x)=b \rightarrow a$,

(5) for any $x \in X,(a \rightarrow x) \rightarrow b=(b \rightarrow x) \rightarrow a$,

(6) for any $x \in X,(a \rightarrow x) \rightarrow(y \rightarrow b)=(b \rightarrow x) \rightarrow(y \rightarrow a)$.

Definition 8. Let $(X ; \rightarrow, 1)$ be a CI-algebra. If for any $x \in X, x \rightarrow 1=x$, then $(X ; \rightarrow, 1)$ is said to be a strong singular.

Proposition 1. If $(X ; \rightarrow, 1)$ is a strong singular CI-algebra. Then $(X ; \rightarrow, 1)$ is a singular CI-algebra.

Proof. For any $x \in X$, assume that $a \rightarrow x=1$, where $a \in X$. By Definition 8 , we have $x \rightarrow 1=x, a \rightarrow 1=$ a. Hence, applying Definition 3,

$$
a=a \rightarrow 1=a \rightarrow(x \rightarrow x)=x \rightarrow(a \rightarrow x)=x \rightarrow 1=x .
$$

By Definition $7, x$ is an atom. Therefore, $(X ; \rightarrow, 1)$ is singular CI-algebra.

Proposition 2. Let $(X ; \rightarrow, 1)$ be a CI-algebra. Then $(X ; \rightarrow, 1)$ is a strong singular CI-algebra if, and only if, $(X ; \rightarrow, 1)$ is an associative BCI-algebra.

Proof. Obviously, every associative BCI-algebra is a strong singular CI-algebra (see [36] and Proposition 1 in Ref. [12]).

Assume that $(X ; \rightarrow, 1)$ is a strong singular CI-algebra.

(1) For any $x, y \in X$, if $x \rightarrow y=y \rightarrow x=1$, then, by Definitions 8 and 3, we have the following:

$$
x=x \rightarrow 1=x \rightarrow(y \rightarrow x)=y \rightarrow(x \rightarrow x)=y \rightarrow 1=y .
$$

(2) For any $x, y, z \in X$, by Proposition 1 and Lemma 2 (4), we can get the following:

$$
(y \rightarrow z) \rightarrow((z \rightarrow x) \rightarrow(y \rightarrow x))=(y \rightarrow z) \rightarrow(y \rightarrow z)=1
$$

Combining Proof (1) and (2), we know that $(X ; \rightarrow, 1)$ is a BCI-algebra. From this, applying Definition 8 and Proposition 1 in Ref. [12], $(X ; \rightarrow, 1)$ is an associative BCI-algebra.

Theorem 4. Let $(X ; \rightarrow, 1)$ be a CI-algebra. Then, $(X, \rightarrow)$ is a (l-l)-quasi neutrosophic triplet loop. Moreover, $(X, \rightarrow)$ is a neutrosophic triplet group if, and only if, $(X ; \rightarrow, 1)$ is a strong singular CI-algebra (associative BCI-algebra). 
Proof. It is similar to the proof of Theorem 3, and we know that $(X, \rightarrow)$ is a $(l-l)$-quasi neutrosophic triplet loop.

If $(X ; \rightarrow, 1)$ is a strong singular CI-algebra, using Proposition $2,(X ; \rightarrow, 1)$ is an associative BCI-algebra. Hence, $\rightarrow$ is associative and commutative, it follows that $(X, \rightarrow)$ is a neutrosophic triplet group.

Conversely, if $(X, \rightarrow)$ is a neutrosophic triplet group, then $\rightarrow$ is associative, thus

$$
x \rightarrow 1=x \rightarrow(x \rightarrow x)=(x \rightarrow x) \rightarrow x=1 \rightarrow x=x .
$$

By Definition 8 we know that $(X ; \rightarrow, 1)$ is a strong singular CI-algebra.

Example 6. Let $X=\{a, b, c, d, e, 1\}$. Define operation $\rightarrow$ on $X$, as following Table 6 . Then, $(X ; \rightarrow, 1)$ is a CI-algebra, and $(X, \rightarrow)$ is a (l-l)-quasi neutrosophic triplet loop, such that

$$
\begin{aligned}
& \left\{\operatorname{neut}_{(l-l)}(a)\right\}=\{1\},\left\{\operatorname{anti}_{(l-l)}(a)\right\}=\{a, b\} ;\left\{\text { neut }_{(l-l)}(b)\right\}=\{1\},\left\{\operatorname{anti}_{(l-l)}(b)\right\}=\{a, b, c\} ; \\
& \left\{\operatorname{neut}_{((-l))}(c)\right\}=\{1\},\left\{\operatorname{anti}_{(-l) l}(c)\right\}=\{c, d, e\} ;\left\{\text { neut }_{(l-l)}(d)\right\}=\{1\},\left\{\operatorname{anti}_{(l-l)}(d)\right\}=\{d, e\} ; \\
& \left\{\operatorname{neut}_{((-l))}(e)\right\}=\{1\},\left\{\operatorname{anti}_{((-l))}(e)\right\}=\{d, e\} ;\left\{\text { neut }_{(I-l)}(1)\right\}=\{1\},\left\{\operatorname{anti}_{(-l)}(1)\right\}=\{1\} .
\end{aligned}
$$

Table 6. CI-algebra and (l-l)-quasi neutrosophic triplet loop.

\begin{tabular}{lllllll}
\hline$\rightarrow$ & $\boldsymbol{a}$ & $\boldsymbol{b}$ & $\boldsymbol{c}$ & $\boldsymbol{d}$ & $\boldsymbol{e}$ & $\mathbf{1}$ \\
\hline $\boldsymbol{a}$ & 1 & 1 & $c$ & $c$ & $c$ & 1 \\
$\boldsymbol{b}$ & 1 & 1 & $c$ & $c$ & $c$ & 1 \\
$\boldsymbol{c}$ & $d$ & 1 & 1 & $a$ & $b$ & $c$ \\
$\boldsymbol{d}$ & $c$ & $c$ & 1 & 1 & 1 & $c$ \\
$\boldsymbol{e}$ & $c$ & $c$ & 1 & 1 & 1 & $c$ \\
$\mathbf{1}$ & $a$ & $b$ & $c$ & $d$ & $e$ & 1 \\
\hline
\end{tabular}

\subsection{BE-Algebras (CI-Algebras) and Their Adjoint Semi-Groups}

I. Fleischer [16] studied the relationship between BCK-algebras and semigroups, and W. Huang [17] studied the close connection between the BCI-algebras and semigroups. In this section, we have studied the adjoint semigroups of the BE-algebras and CI-algebras, and will give some interesting examples.

For any BE-algebra or CI-algebra $(X ; \rightarrow, 1)$, and any element $a$ in $X$, we use $p_{a}$ to denote the self-map of $X$ defined by the following:

$$
p_{a}: X \rightarrow X ; \mapsto a \rightarrow x \text {, for all } x \in X
$$

Theorem 5. Let $(X ; \rightarrow, 1)$ be a BE-algebra (or CI-algebra), and $M(X)$ be the set of finite products $p_{a}{ }^{*} \ldots{ }^{*} p_{b}$ of self-map of $X$ with $a, \ldots, b \in X$, where * represents the composition operation of mappings. Then $(M(X), *)$ is a commutative semigroup with identity $p_{1}$.

Proof. Since the composition operation of mappings satisfies the associative law, $\left(M(X),{ }^{*}\right)$ is a semigroup. Moreover, since

$$
p_{1}: X \rightarrow X \mapsto 1 \rightarrow x \text {, for all } x \in X .
$$

Applying Definition 3 (ii), we get that $p_{1}(x)=x$ for any $x \in X$. Hence, $p_{1}{ }^{*} m=p_{1}{ }^{*} m=m$ for any $m \in M(X)$. 
For any $a, b \in X$, using Definition 3 (iii) we have $(\forall x \in X)$ the following:

$$
\left(p_{a}^{*} p_{b}\right)(x)=p_{a}(b \rightarrow x)=a \rightarrow(b \rightarrow x)=b \rightarrow(a \rightarrow x)=p_{b}(a \rightarrow x)=\left(p_{b}^{*} p_{a}\right)(x) .
$$

Therefore, $\left(M(X),{ }^{*}\right)$ is a commutative semigroup with identity $p_{1}$.

Now, we call $\left(M(X),{ }^{*}\right)$ the adjoint semigroup of $X$.

Example 7. Let $X=\{a, b, c, 1\}$. Define operation $\rightarrow$ on $X$, as following Table 7 . Then, $(X ; \rightarrow, 1)$ is a $B E$-algebra, and

$p_{a}: X \rightarrow X ; a \mapsto 1, b \mapsto 1, c \mapsto 1,1 \mapsto 1$. It is abbreviated to $p_{a}=(1,1,1,1)$.

$p_{b}: X \rightarrow X ; a \mapsto c, b \mapsto 1, c \mapsto a, 1 \mapsto 1$. It is abbreviated to $p_{b}=(c, 1, a, 1)$.

$p_{c}: X \rightarrow X ; a \mapsto 1, b \mapsto 1, c \mapsto 1,1 \mapsto 1$. It is abbreviated to $p_{c}=(1,1,1,1)$.

$p_{1}: X \rightarrow X ; a \mapsto a, b \mapsto b, c \mapsto c, 1 \mapsto 1$. It is abbreviated to $p_{1}=(a, b, c, 1)$.

We can verify that $p_{a} * p_{a}=p_{a}, p_{a} * p_{b}=p_{a}, p_{a} * p_{c}=p_{a} ; p_{b} * p_{b}=(a, 1, c, 1), p_{b} * p_{c}=p_{c}=p_{a} ; p_{a}$ * $\left(p_{b} * p_{b}\right)=p_{a}, p_{b} *\left(p_{b} * p_{b}\right)=p_{b}, p_{c}^{*}\left(p_{b} * p_{b}\right)=p_{c}=p_{a}$. Denote $p_{b b}=p_{b}^{*} p_{b}=(a, 1, c, 1)$, then $M(X)=\left\{p_{a}\right.$, $\left.p_{b}, p_{b b}, p_{1}\right\}$, and its Cayley table is Table 8 . Obviously, $\left(M(X),{ }^{*}\right)$ is a commutative neutrosophic triplet group and

$\operatorname{neut}\left(p_{a}\right)=p_{a}$, anti $\left(p_{a}\right)=p_{a} ; \operatorname{neut}\left(p_{b}\right)=p_{b b}$, anti $\left(p_{b}\right)=p_{b} ; \operatorname{neut}\left(p_{b b}\right)=p_{b b}$, anti $\left(p_{b b}\right)=p_{b b} ; \operatorname{neut}\left(p_{1}\right)=p_{1}, \operatorname{anti}\left(p_{1}\right)=p_{1}$.

Table 7. BE-algebra.

\begin{tabular}{ccccc}
\hline$\rightarrow$ & $\boldsymbol{a}$ & $\boldsymbol{b}$ & $\boldsymbol{c}$ & $\mathbf{1}$ \\
\hline $\boldsymbol{a}$ & 1 & 1 & 1 & 1 \\
$\boldsymbol{b}$ & $c$ & 1 & $a$ & 1 \\
$c$ & 1 & 1 & 1 & 1 \\
$\mathbf{1}$ & $a$ & $b$ & $c$ & 1 \\
\hline
\end{tabular}

Table 8. Adjoint semigroup of the above BE-algebra.

\begin{tabular}{ccccc}
\hline$*$ & $p_{\boldsymbol{a}}$ & $p_{\boldsymbol{b}}$ & $p_{\boldsymbol{b} \boldsymbol{b}}$ & $p_{\boldsymbol{1}}$ \\
\hline$p_{\boldsymbol{a}}$ & $p_{a}$ & $p_{a}$ & $p_{a}$ & $p_{a}$ \\
$p_{\boldsymbol{b}}$ & $p_{a}$ & $p_{b b}$ & $p_{b}$ & $p_{b}$ \\
$p_{\boldsymbol{b} \boldsymbol{b}}$ & $p_{a}$ & $p_{b}$ & $p_{b b}$ & $p_{b b}$ \\
$p_{\mathbf{1}}$ & $p_{a}$ & $p_{b}$ & $p_{b b}$ & $p_{1}$ \\
\hline
\end{tabular}

Example 8. Let $X=\{a, b, 1\}$. Define operation $\rightarrow$ on $X$, as following Table 9. Then, $(X ; \rightarrow, 1)$ is a CI-algebra, and $p_{a}: X \rightarrow X ; a \mapsto 1, b \mapsto a, 1 \mapsto b$. It is abbreviated to $p_{a}=(1, a, b)$.

$p_{b}: X \rightarrow X ; a \mapsto b, b \mapsto 1,1 \mapsto a$. It is abbreviated to $p_{b}=(b, 1, a)$.

$p_{1}: X \rightarrow X ; a \mapsto a, b \mapsto b, 1 \mapsto 1$. It is abbreviated to $p_{1}=(a, b, 1)$.

We can verify that $p_{a} * p_{a}=p_{b}, p_{a} * p_{b}=p_{1} ; p_{b} * p_{b}=p_{a}$. Then $M(X)=\left\{p_{a}, p_{b}, p_{1}\right\}$ and its Cayley table is Table 10. Obviously, $\left(M(X),{ }^{*}\right)$ is a commutative group with identity $p_{1}$ and $\left(p_{a}\right)^{-1}=p_{b},\left(p_{b}\right)^{-1}=p_{a}$. 
Table 9. CI-algebra.

\begin{tabular}{cccc}
\hline$\rightarrow$ & $\boldsymbol{a}$ & $\boldsymbol{b}$ & $\mathbf{1}$ \\
\hline $\boldsymbol{a}$ & 1 & $a$ & $b$ \\
$\boldsymbol{b}$ & $b$ & 1 & $a$ \\
$\mathbf{1}$ & $a$ & $b$ & 1 \\
\hline
\end{tabular}

Table 10. Adjoint semigroup of the above CI-algebra.

\begin{tabular}{llll}
\hline$*$ & $p_{\boldsymbol{a}}$ & $p_{\boldsymbol{b}}$ & $p_{\mathbf{1}}$ \\
\hline$p_{\boldsymbol{a}}$ & $p_{b}$ & $p_{1}$ & $p_{a}$ \\
$p_{\boldsymbol{b}}$ & $p_{1}$ & $p_{a}$ & $p_{b}$ \\
$p_{\mathbf{1}}$ & $p_{a}$ & $p_{b}$ & $p_{1}$ \\
\hline
\end{tabular}

Theorem 6. Let $(X ; \rightarrow, 1)$ be a singular CI-algebra, and $M(X)$ be the adjoint semigroup. Then $\left(M(X),{ }^{*}\right)$ is a commutative group with identity $p_{1}$, where $M(X)=\left\{p_{a} \mid a \in X\right\}$ and $|M(X)|=|X|$.

Proof. (1) First, we prove that for any singular CI-algebra, $a \rightarrow(b \rightarrow x)=((a \rightarrow 1) \rightarrow b) \rightarrow x, \forall a, b$, $x \in X$.

In fact, by Definition 7 and Lemma 2, we have the following:

$$
\begin{aligned}
((a \rightarrow 1) \rightarrow b) \rightarrow x & =((a \rightarrow 1) \rightarrow b) \rightarrow((x \rightarrow 1) \rightarrow 1) \\
& =(x \rightarrow 1) \rightarrow(((a \rightarrow 1) \rightarrow b) \rightarrow 1) \\
& =(x \rightarrow 1) \rightarrow(((a \rightarrow 1) \rightarrow 1) \rightarrow(b \rightarrow 1)) \\
& =(x \rightarrow 1) \rightarrow(a \rightarrow(b \rightarrow 1)) \\
& =a \rightarrow((x \rightarrow 1) \rightarrow(b \rightarrow 1)) \\
& =a \rightarrow(b \rightarrow x) .
\end{aligned}
$$

(2) Second, we prove that for any singular CI-algebra, $a \neq b \Rightarrow p_{a} \neq p_{b}, \forall a, b \in X$.

Assume $p_{a}=p_{b}, a, b \in X$. Then, for all $x$ in $X, p_{a}(x)=p_{b}(x)$. Hence,

$$
a \rightarrow b=p_{a}(b)=p_{b}(b)=b \rightarrow b=1 .
$$

From this, applying Lemma 2 (1) and (6) we get

$$
a=(a \rightarrow 1) \rightarrow 1=(a \rightarrow 1) \rightarrow(a \rightarrow b)=(b \rightarrow 1) \rightarrow(a \rightarrow a)=(b \rightarrow 1) \rightarrow 1=b .
$$

(3) Using Lemma 2 (1), we know that for any $a, b \in X$, there exist $c \in X$, such that $p_{a} * p_{b}=p_{c}$, where $c=(a \rightarrow 1) \rightarrow b$. This means that $M(X) \subseteq\left\{p_{a} \mid a \in X\right\}$. By the definition of $M(X),\left\{p_{a} a \in X\right\} \subseteq$ $M(X)$. Hence, $M(X)=\left\{p_{a} \mid a \in X\right\}$.

(4) Using Lemma 2 (2) and (3), we know that $|M(X)|=|X|$.

\section{Quasi Neutrosophic Triplet Structures in Pseudo BE-Algebras and Pseudo CI-Algebras}

Like the above Section 4, we can discuss the relationships between pseudo BE-algebras (pseudo CI-algebras) and quasi neutrosophic triplet structures. This section will give some related results and examples, but part of the simple proofs will be omitted. 


\subsection{Pseudo BE-Algebras (Pseudo CI-Algebras) and (l-l)-Quasi Neutrosophic Triplet Loops}

Theorem 7. Let $(X ; \rightarrow, \rightsquigarrow, 1)$ be pseudo BE-algebra. Then $(X, \rightarrow)$ and $(X, \rightsquigarrow)$ are (l-l)-quasi neutrosophic triplet loops. And, when $|X|>1,(X, \rightarrow)$ and $(X, \rightsquigarrow)$ are not (lr-l)-quasi neutrosophic triplet loops with neutral element 1.

Example 9. Let $X=\{a, b, c, 1\}$. Define operations $\rightarrow$ and $\rightsquigarrow$ on $X$ as following Tables 11 and 12. Then, $(X ; \rightarrow$, $\rightsquigarrow, 1)$ is a pseudo BE-algebra, and $(X, \rightarrow)$ and $(X, \rightsquigarrow)$ are (l-l)-quasi neutrosophic triplet loops.

Table 11. Pseudo BE-algebra (1).

\begin{tabular}{ccccc}
\hline$\rightarrow$ & $\boldsymbol{a}$ & $\boldsymbol{b}$ & $\boldsymbol{c}$ & $\mathbf{1}$ \\
\hline $\boldsymbol{a}$ & 1 & 1 & $b$ & 1 \\
$\boldsymbol{b}$ & $a$ & 1 & $c$ & 1 \\
$c$ & 1 & 1 & 1 & 1 \\
$\mathbf{1}$ & $a$ & $b$ & $c$ & 1 \\
\hline
\end{tabular}

Table 12. Pseudo BE-algebra (2).

\begin{tabular}{lllll}
\hline$\rightsquigarrow$ & $\boldsymbol{a}$ & $\boldsymbol{b}$ & $\boldsymbol{c}$ & $\mathbf{1}$ \\
\hline $\boldsymbol{a}$ & 1 & 1 & $a$ & 1 \\
$\boldsymbol{b}$ & $a$ & 1 & $a$ & 1 \\
$\boldsymbol{c}$ & 1 & 1 & 1 & 1 \\
$\mathbf{1}$ & $a$ & $b$ & $c$ & 1 \\
\hline
\end{tabular}

Definition 9. $([44,46])$ Let a be an element of a pseudo CI-algebra $(X ; \rightarrow, \rightsquigarrow, 1)$. $a$ is said to be an atom in $X$ if for any $x \in X, a \rightarrow x=1$ implies $a=x$.

Applying the results in Ref. [44-46] we have the following propositions (the proofs are omitted).

Proposition 3. If $(X ; \rightarrow, \rightsquigarrow, 1)$ is a pseudo CI-algebra, then for all $x, y \in X$

(1) $x \leq(x \rightarrow y) \rightsquigarrow y, x \leq(x \rightsquigarrow y) \rightarrow y$,

(2) $x \leq y \rightarrow z \Leftrightarrow y \leq x \rightsquigarrow z$,

(3) $(x \rightarrow y) \rightarrow 1=(x \rightarrow 1) \rightsquigarrow(y \rightsquigarrow 1),(x \rightsquigarrow y) \rightsquigarrow 1=(x \rightsquigarrow 1) \rightarrow(y \rightarrow 1)$,

(4) $x \rightarrow 1=x \rightsquigarrow 1$,

(5) $x \leq y$ implies $x \rightarrow 1=y \rightarrow 1$.

Proposition 4. Let $(X ; \rightarrow, \rightsquigarrow, 1)$ be a pseudo CI-algebra. If $a, b \in X$ are atoms in $X$, then the following are true:

(1) $a=(a \rightarrow 1) \rightarrow 1$,

(2) for any $x \in X,(a \rightarrow x) \rightsquigarrow x=a,(a \rightsquigarrow x) \rightarrow x=a$,

(3) for any $x \in X,(a \rightarrow x) \rightsquigarrow 1=x \rightarrow a,(a \rightsquigarrow x) \rightarrow 1=x \rightsquigarrow a$,

(4) for any $x \in X, x \rightarrow a=(a \rightarrow 1) \rightsquigarrow(x \rightarrow 1), x \rightsquigarrow a=(a \rightsquigarrow 1) \rightarrow(x \rightsquigarrow 1)$.

Definition 10. A pseudo CI-algebra $(X ; \rightarrow, \rightsquigarrow, 1)$ is said to be singular if every element of $X$ is an atom. A pseudo CI-algebra $(X ; \rightarrow, \rightsquigarrow, 1)$ is said to be strong singular if for any $x \in X, x \rightarrow 1=x=x \rightsquigarrow 1$.

Proposition 5. If $(X ; \rightarrow, \rightsquigarrow, 1)$ is a strong singular pseudo CI-algebra. Then $(X ; \rightarrow, \rightsquigarrow, 1)$ is singular. 
Proof. For any $x \in X$, assume that $a \rightarrow x=1$, where $a \in X$. It follows from Definition 10,

$$
x \rightarrow 1=x=x \rightsquigarrow 1, a \rightarrow 1=a=a \rightsquigarrow 1 .
$$

Hence, applying Definition 4 and Proposition 3,

$$
a=a \rightarrow 1=a \rightarrow(x \rightsquigarrow x)=x \rightsquigarrow(a \rightarrow x)=x \rightsquigarrow 1=x .
$$

By Definition $9, x$ is an atom. Therefore, $(X ; \rightarrow, \rightsquigarrow, 1)$ is singular pseudo CI-algebra.

Applying Theorem 3.11 in Ref. [46], we can get the following:

Lemma 3. Let $(X ; \rightarrow, \rightsquigarrow, 1)$ be a pseudo CI-algebra. Then the following statements are equivalent:

(1) $x \rightarrow(y \rightarrow z)=(x \rightarrow y) \rightarrow z$, for all $x, y$, $z$ in $X$;

(2) $x \rightarrow 1=x=x \rightsquigarrow 1$, for every $x$ in $X$;

(3) $x \rightarrow y=x \rightsquigarrow y=y \rightarrow x$, for all $x$, $y$ in $X$;

(4) $x \rightsquigarrow(y \rightsquigarrow z)=(x \rightsquigarrow y) \rightsquigarrow z$, for all $x, y, z$ in $X$.

Proposition 6. Let $(X ; \rightarrow, \rightsquigarrow, 1)$ be a pseudo CI-algebra. Then $(X ; \rightarrow, \rightsquigarrow, 1)$ is a strong singular pseudo CI-algebra if, and only if, $\rightarrow=\rightsquigarrow$ and $(X ; \rightarrow, 1)$ is an associative BCI-algebra.

Proof. We know that every associative BCI-algebra is a strong singular pseudo CI-algebra.

Now, suppose that $(X ; \rightarrow, 1)$ is a strong singular pseudo CI-algebra. By Definition 10 and Lemma 3 (3), $x \rightarrow y=x \rightsquigarrow y, \forall x, y \in X$. That is, $\rightarrow=\rightsquigarrow$. Hence, $(X ; \rightarrow, 1)$ is a strong singular CI-algebra. It follows that $(X ; \rightarrow, 1)$ is an associative BCI-algebra (using Proposition 2).

Theorem 8. Let $(X ; \rightarrow, \rightsquigarrow, 1)$ be a pseudo CI-algebra. Then $(X, \rightarrow)$ and $(X, \rightsquigarrow)$ are(l-l)-quasi neutrosophic triplet loops. Moreover, $(X, \rightarrow)$ and $(X, \rightsquigarrow)$ are neutrosophic triplet groups if, and only if, $(X ; \rightarrow, \rightsquigarrow, 1)$ is a strong singular pseudo CI-algebra (associative BCI-algebra).

Proof. Applying Lemma 3, and the proof is omitted.

\subsection{Pseudo BE-Algebras (Pseudo CI-Algebras) and Their Adjoint Semi-Groups}

For any pseudo BE-algebra or pseudo CI-algebra $(X ; \rightarrow, \rightsquigarrow, 1)$ as well as any element $a$ in $X$, we use $p_{a} \rightarrow$ and $p_{a} \rightsquigarrow$ to denote the self-map of $X$, which is defined by the following:

$$
\begin{aligned}
& p_{a} \rightarrow: X \rightarrow X ; \mapsto a \rightarrow x, \text { for all } x \in X . \\
& p_{a}{ }^{\rightsquigarrow}: X \rightarrow X ; \mapsto a \rightsquigarrow x, \text { for all } x \in X .
\end{aligned}
$$

Theorem 9. Let $(X ; \rightarrow, \rightsquigarrow, 1)$ be a pseudo BE-algebra (or pseudo CI-algebra), and

$$
\begin{gathered}
M^{\rightarrow}(X)=\left\{\text { finite products } p_{a} \rightarrow * \ldots * p_{b} \rightarrow \text { of self-map of } X \mid a, \ldots, b \in X\right\}, \\
M^{\rightsquigarrow}(X)=\left\{\text { finite products } p_{a}{ }^{\cdots} \ldots{ }^{*} p_{b} \rightsquigarrow \text { of self-map of } X \mid a, \ldots, b \in X\right\}, \\
M(X)=\left\{\text { finite products } p_{a} \rightarrow\left(\text { or } p_{a}{ }^{\rightsquigarrow}\right){ }^{*} \ldots{ }^{*} p_{b} \rightarrow\left(\text { or } p_{b}{ }^{\rightsquigarrow}\right) \text { of self-map of } X \mid a, \ldots, b \in X\right\},
\end{gathered}
$$

where * represents the composition operation of mappings. Then $\left(M^{\rightarrow}(X),{ }^{*}\right),\left(M^{\rightsquigarrow}(X),{ }^{*}\right)$, and $\left(M(X),{ }^{*}\right)$ are all semigroups with the identity $p_{1}=p_{1} \rightarrow=p_{1}$. 
Proof. It is similar to Theorem 5.

Now, we call $\left(M^{\rightarrow}(X),{ }^{*}\right),\left(M^{\rightsquigarrow}(X),{ }^{*}\right)$, and $\left(M(X),{ }^{*}\right)$ the adjoint semigroups of $X$.

Example 10. Let $X=\{a, b, c, 1\}$. Define operations $\rightarrow$ and $\rightsquigarrow$ on $X$ as following Tables 13 and 14. Then, (X; $\rightarrow, \rightsquigarrow, 1)$ is a pseudo BE-algebra, and

$$
p_{a} \rightarrow=(1, b, b, 1), p_{b} \rightarrow=(a, 1, c, 1), p_{c} \rightarrow=(1,1,1,1), p_{1} \rightarrow=(a, b, c, 1) .
$$

We can verify the following:

$$
\begin{gathered}
p_{a} \rightarrow * p_{a} \rightarrow=p_{a} \rightarrow, p_{a} \rightarrow * p_{b} \rightarrow=(1,1, b, 1), p_{a} \rightarrow * p_{c} \rightarrow=p_{c} \rightarrow, p_{a} \rightarrow * p_{1} \rightarrow=p_{a} \rightarrow ; \\
p_{b} \rightarrow * p_{a} \rightarrow=p_{c} \rightarrow, p_{b} \rightarrow * p_{b} \rightarrow=p_{b} \rightarrow, p_{b} \rightarrow * p_{c} \rightarrow=p_{c} \rightarrow, p_{b} \rightarrow * p_{1} \rightarrow=p_{b} \rightarrow ; \\
p_{c} \rightarrow * p_{a} \rightarrow=p_{c} \rightarrow, p_{c} \rightarrow * p_{b} \rightarrow=p_{c} \rightarrow, p_{c} \rightarrow * p_{c} \rightarrow=p_{c} \rightarrow, p_{c} \rightarrow * p_{1} \rightarrow=p_{c} \rightarrow ; \\
p_{1} \rightarrow * p_{a} \rightarrow=p_{a} \rightarrow, p_{1} \rightarrow * p_{b} \rightarrow=p_{b} \rightarrow, p_{1} \rightarrow * p_{c} \rightarrow=p_{c} \rightarrow, p_{1} \rightarrow * p_{1} \rightarrow=p_{1} \rightarrow .
\end{gathered}
$$

Denote $p_{a b} \rightarrow=p_{a} \rightarrow * p_{b} \rightarrow=(1,1, b, 1)$, then $p_{a b} \rightarrow * p_{a} \rightarrow=p_{c} \rightarrow, p_{a b} \rightarrow * p_{b} \rightarrow=p_{a b} \rightarrow, p_{a b} \rightarrow * p_{a b} \rightarrow=$ $p^{\rightarrow}, p_{a b} \rightarrow * p_{c} \rightarrow=p_{c} \rightarrow$. Hence, $M^{\rightarrow}(X)=\left\{p_{a} \rightarrow, p_{b} \rightarrow, p_{a b} \rightarrow, p_{c} \rightarrow, p_{1} \rightarrow\right\}$ and its Cayley table is Table 15 . Obviously, $\left(M^{\rightarrow}(X),{ }^{*}\right)$ is a non-commutative semigroup, but it is not a neutrosophic triplet group.

Table 13. Pseudo BE-algebra and adjoint semigroups (1).

\begin{tabular}{ccccc}
\hline$\rightarrow$ & $\boldsymbol{a}$ & $\boldsymbol{b}$ & $\boldsymbol{c}$ & $\mathbf{1}$ \\
\hline $\boldsymbol{a}$ & 1 & $b$ & $b$ & 1 \\
$\boldsymbol{b}$ & $a$ & 1 & $c$ & 1 \\
$c$ & 1 & 1 & 1 & 1 \\
$\mathbf{1}$ & $a$ & $b$ & $c$ & 1 \\
\hline
\end{tabular}

Table 14. Pseudo BE-algebra and adjoint semigroups (2).

\begin{tabular}{lllll}
\hline$\rightsquigarrow$ & $\boldsymbol{a}$ & $\boldsymbol{b}$ & $\boldsymbol{c}$ & $\mathbf{1}$ \\
\hline $\boldsymbol{a}$ & 1 & $b$ & $c$ & 1 \\
$\boldsymbol{b}$ & $a$ & 1 & $a$ & 1 \\
$c$ & 1 & 1 & 1 & 1 \\
$\mathbf{1}$ & $a$ & $b$ & $c$ & 1 \\
\hline
\end{tabular}

Table 15. Pseudo BE-algebra and adjoint semigroups (3).

\begin{tabular}{clllll}
\hline$*$ & $p_{\boldsymbol{a}} \rightarrow$ & $p_{\boldsymbol{b}} \rightarrow$ & $p_{\boldsymbol{a b}} \rightarrow$ & $p_{c} \rightarrow$ & $p_{\mathbf{1}} \rightarrow$ \\
\hline$p_{\boldsymbol{a}} \rightarrow$ & $p_{a} \rightarrow$ & $p_{a b} \rightarrow$ & $p_{a b} \rightarrow$ & $p_{c} \rightarrow$ & $p_{a} \rightarrow$ \\
$p_{\boldsymbol{b}} \rightarrow$ & $p_{c} \rightarrow$ & $p_{b} \rightarrow$ & $p_{c} \rightarrow$ & $p_{c} \rightarrow$ & $p_{b} \rightarrow$ \\
$p_{a b} \rightarrow$ & $p_{c} \rightarrow$ & $p_{a b} \rightarrow$ & $p_{c} \rightarrow$ & $p_{c} \rightarrow$ & $p_{a b} \rightarrow$ \\
$p_{c} \rightarrow$ & $p_{c} \rightarrow$ & $p_{c} \rightarrow$ & $p_{c} \rightarrow$ & $p_{c} \rightarrow$ & $p_{c} \rightarrow$ \\
$p_{\mathbf{1}} \rightarrow$ & $p_{a} \rightarrow$ & $p_{b} \rightarrow$ & $p_{a b} \rightarrow$ & $p_{c} \rightarrow$ & $p_{1} \rightarrow$ \\
\hline
\end{tabular}

Similarly, we can verify that

$$
\begin{aligned}
& p_{a}=(1, b, c, 1), p_{b}^{\rightsquigarrow}=(a, 1, a, 1), p_{c}^{\rightsquigarrow}=(1,1,1,1), p_{1}^{\rightsquigarrow}=(a, b, c, 1) .
\end{aligned}
$$

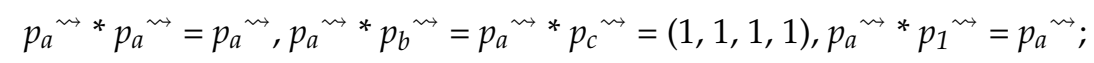

$$
\begin{aligned}
& p_{b}{ }^{*} p_{a} \rightsquigarrow(1,1, a, 1), p_{b} \rightsquigarrow * p_{b} \rightsquigarrow=p_{b}{ }^{\rightsquigarrow}, p_{b}{ }^{*} p_{c}{ }^{\rightsquigarrow}=p_{c}{ }^{\rightsquigarrow}, p_{b}{ }^{*} p_{1} \rightsquigarrow=p_{b}{ }^{\rightsquigarrow} \text {; }
\end{aligned}
$$




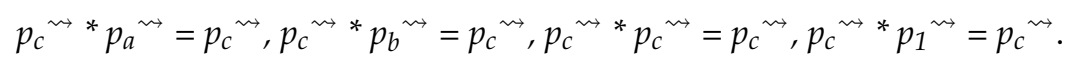

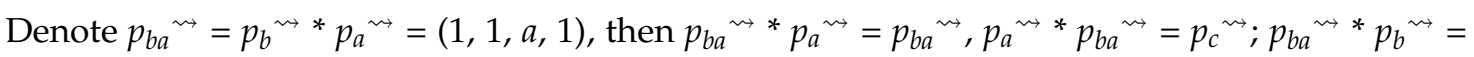

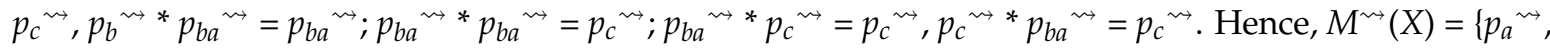
$\left.p_{b}{ }^{\rightsquigarrow}, p_{b a}{ }^{\rightsquigarrow}, p_{c}{ }^{\rightsquigarrow}, p_{1}{ }^{\rightsquigarrow}\right\}$ and its Cayley table is Table 16. Obviously, $\left(M^{\rightsquigarrow}(X),{ }^{*}\right)$ is a non-commutative semigroup, but it is not a neutrosophic triplet group.

Table 16. Pseudo BE-algebra and adjoint semigroups (4).

\begin{tabular}{|c|c|c|c|c|c|}
\hline$*$ & $p_{a}^{\rightsquigarrow}$ & $p_{b}^{\rightsquigarrow}$ & $p_{b a} \rightsquigarrow$ & $p_{c}^{\rightsquigarrow}$ & $p_{1} \rightsquigarrow$ \\
\hline$p_{a}^{\rightsquigarrow}$ & $p_{a} \rightsquigarrow$ & $p_{c} \rightsquigarrow$ & $p_{c} \rightsquigarrow$ & $p_{c} \rightsquigarrow$ & $p_{a} \rightsquigarrow$ \\
\hline$p_{b}^{\rightsquigarrow}$ & $p_{b a} \rightsquigarrow$ & $p_{b}^{\rightsquigarrow}$ & $p_{b a} \rightsquigarrow$ & $p_{c}^{\rightsquigarrow}$ & $p_{b} \rightsquigarrow$ \\
\hline$p_{b a}$ & $p_{b a} \rightsquigarrow$ & $p_{c}^{\rightsquigarrow}$ & $p_{c}^{\rightsquigarrow}$ & $p_{c} \rightsquigarrow$ & $p_{b a} \rightsquigarrow$ \\
\hline$p_{c}^{\rightsquigarrow}$ & $p_{c}^{\rightsquigarrow}$ & $p_{c}^{\rightsquigarrow}$ & $p_{c}^{\rightsquigarrow}$ & $p_{c} \rightsquigarrow$ & $p_{c} \rightsquigarrow$ \\
\hline$p_{1}^{\rightsquigarrow}$ & $p_{a} \rightsquigarrow$ & $p_{b}$ & $p_{b a} \rightsquigarrow$ & $p_{c}^{\rightsquigarrow}$ & $p_{1}^{\rightsquigarrow}$ \\
\hline
\end{tabular}

Now, we consider $M(X)$. Since

$$
\begin{aligned}
& p_{c} \rightarrow=(1,1,1,1)=p_{c}{ }^{\rightsquigarrow}, p_{1} \rightarrow=(a, b, c, 1)=p_{1} \rightsquigarrow ; \\
& p_{a} \rightarrow * p_{a}=p_{a} \rightarrow, p_{a} * * p_{a} \rightarrow=p_{a} \rightarrow \text {; } \\
& p_{a} \rightarrow * p_{b}=(1,1,1,1)=p_{c} \rightarrow, p_{b} * * p_{a} \rightarrow=(1,1,1,1)=p_{c} \rightarrow \text {; } \\
& p_{a}{ }^{\rightsquigarrow *} p_{b} \rightarrow=p_{b} \rightarrow * p_{a}{ }^{\rightsquigarrow}=(1,1, c, 1) \text {; } \\
& p_{a} \rightsquigarrow * p_{a b} \rightarrow=p_{a b} \rightarrow, p_{a b} \rightarrow * p_{a}{ }^{\rightsquigarrow}=p_{a b} \rightarrow ; p_{b} \rightarrow * p_{b}{ }^{\rightsquigarrow}=p_{b} \text { }, p_{b}{ }^{* *} p_{b} \rightarrow=p_{b} \text {; } \\
& p_{a b} \rightarrow * p_{b}{ }^{\rightsquigarrow}=(1,1,1,1)=p_{c} \rightarrow, p_{b}{ }^{\star *} p_{a b} \rightarrow=(1,1,1,1)=p_{c} \rightarrow \text {; } \\
& p_{a} \rightarrow * p_{b a} \rightsquigarrow=(1,1,1,1)=p_{c} \rightarrow, p_{b a}{ }^{\rightsquigarrow *} p_{a} \rightarrow=(1,1,1,1)=p_{c} \rightarrow \text {; } \\
& p_{b} \rightarrow * p_{b a}=p_{b a}{ }^{\rightsquigarrow}, p_{b a}{ }^{*} p_{b} \rightarrow=p_{b a} \rightsquigarrow \text {; } \\
& p_{a b} \rightarrow * p_{b a}{ }^{\rightsquigarrow}=(1,1,1,1)=p_{c} \rightarrow, p_{b a} \rightsquigarrow * p_{a b} \rightarrow=(1,1,1,1)=p_{c} \rightarrow \text {. }
\end{aligned}
$$

\begin{tabular}{|c|c|c|c|c|c|c|c|c|c|}
\hline * & $p_{a} \rightarrow$ & $p_{a}^{\rightsquigarrow}$ & $p_{b} \rightarrow$ & $p_{b}^{\rightsquigarrow}$ & $p_{a b} \rightarrow$ & $p_{b a}{ }^{\rightsquigarrow}$ & $p$ & $p_{c} \rightarrow$ & $p_{1} \rightarrow$ \\
\hline$p_{a} \rightarrow$ & $p_{a} \rightarrow$ & $p_{a} \rightarrow$ & $p_{a b} \rightarrow$ & $p_{c} \rightarrow$ & $p_{a b} \rightarrow$ & $p_{c} \rightarrow$ & $p_{a b} \rightarrow$ & $p_{c} \rightarrow$ & $p_{a} \rightarrow$ \\
\hline$p_{a}^{\rightsquigarrow}$ & $p_{a} \rightarrow$ & $p_{a} \rightsquigarrow$ & $p$ & $p_{c} \rightarrow$ & $p_{a b} \rightarrow$ & $p_{b a}{ }^{\rightsquigarrow}$ & $p$ & $p_{c} \rightarrow$ & $p_{a} \rightsquigarrow$ \\
\hline$p_{b} \rightarrow$ & $p_{c} \rightarrow$ & $p$ & $p_{b} \rightarrow$ & $p_{b}^{\rightsquigarrow}$ & $p_{c} \rightarrow$ & $p_{b a}{ }^{\rightsquigarrow}$ & $p$ & $p_{c} \rightarrow$ & $p_{b} \rightarrow$ \\
\hline$p_{b}^{\rightsquigarrow}$ & $p_{c} \rightarrow$ & $p_{b a} \rightsquigarrow$ & $p_{b} \rightsquigarrow$ & $p_{b}^{\rightsquigarrow}$ & $p_{c} \rightarrow$ & $p_{b a} \rightsquigarrow$ & $p_{b a} \rightsquigarrow$ & $p_{c} \rightarrow$ & $p_{b}^{\rightsquigarrow}$ \\
\hline$p_{a b} \rightarrow$ & $p_{c} \rightarrow$ & $p_{a b} \rightarrow$ & $p_{a b} \rightarrow$ & $p_{c} \rightarrow$ & $p_{c} \rightarrow$ & $p_{c} \rightarrow$ & $p_{a b} \rightarrow$ & $p_{c} \rightarrow$ & $p_{a b} \rightarrow$ \\
\hline$p_{b a}{ }^{\rightsquigarrow}$ & $p_{c} \rightarrow$ & $p_{b a} \rightsquigarrow$ & $p_{b a}{ }^{\rightsquigarrow}$ & $p_{c} \rightarrow$ & $p_{c} \rightarrow$ & $p_{c} \rightarrow$ & $p_{b a} \rightsquigarrow$ & $p_{c} \rightarrow$ & $p_{b a} \rightsquigarrow$ \\
\hline$p$ & $p_{c} \rightarrow$ & $p$ & $p$ & $p_{c} \rightarrow$ & $p_{c} \rightarrow$ & $p_{c} \rightarrow$ & $p$ & $p_{c} \rightarrow$ & $p$ \\
\hline $\overrightarrow{p_{c}} \rightarrow$ & $p_{c} \rightarrow$ & $p_{c} \rightarrow$ & $p_{c} \rightarrow$ & $p_{c} \rightarrow$ & $p_{c} \rightarrow$ & $p_{c} \rightarrow$ & $p_{c} \rightarrow$ & $p_{c} \rightarrow$ & $p_{c} \rightarrow$ \\
\hline$p_{1} \rightarrow$ & $p_{a} \rightarrow$ & $p_{a}^{\rightsquigarrow}$ & $p_{b} \rightarrow$ & $p_{b}^{\rightsquigarrow}$ & $p_{a b} \rightarrow$ & $p_{b a}{ }^{\rightsquigarrow}$ & $p$ & $p_{c} \rightarrow$ & $p_{1} \rightarrow$ \\
\hline
\end{tabular}

Denote $p=(1,1, c, 1)$, then $M(X)=\left\{p_{a} \rightarrow, p_{a} \rightsquigarrow, p_{b} \rightarrow, p_{b}{ }^{\rightsquigarrow}, p_{a b} \rightarrow, p_{b a}{ }^{\rightsquigarrow}, p_{,}, p_{c} \rightarrow, p_{1} \rightarrow\right\}$, and Table 17 is its Cayley table (it is a non-commutative semigroup, but it is not a neutrosophic triplet group).

Table 17. Pseudo BE-algebra and adjoint semigroups (5).

The following example shows that the adjoint semigroups of a pseudo BE-algebra may be a commutative neutrosophic triplet group. 
Example 11. Let $X=\{a, b, c, d, 1\}$. Define operations $\rightarrow$ and $\rightsquigarrow$ on $X$ as Tables 18 and 19. Then, $(X ; \rightarrow, \rightsquigarrow, 1)$ is a pseudo BE-algebra, as well as the following:

$$
p_{a} \rightarrow=(1, c, c, 1,1), p_{b} \rightarrow=(d, 1,1, d, 1), p_{c} \rightarrow=(d, 1,1, d, 1), p_{d} \rightarrow=(1, c, c, 1,1), p_{1} \rightarrow=(a, b, c, d, 1) .
$$

We can verify the following:

$$
\begin{aligned}
& p_{a} \rightarrow * p_{a} \rightarrow=p_{a} \rightarrow, p_{a} \rightarrow * p_{b} \rightarrow=p_{a} \rightarrow * p_{c} \rightarrow=(1,1,1,1,1), p_{a} \rightarrow * p_{d} \rightarrow=p_{a} \rightarrow, p_{a} \rightarrow * p_{1} \rightarrow=p_{a} \rightarrow \text {; } \\
& p_{b} \rightarrow * p_{a} \rightarrow=(1,1,1,1,1), p_{b} \rightarrow * p_{b} \rightarrow=p_{b} \rightarrow * p_{c} \rightarrow=p_{b} \rightarrow, p_{b} \rightarrow * p_{d} \rightarrow=(1,1,1,1,1), p_{b} \rightarrow * p_{1} \rightarrow=p_{b} \rightarrow \text {; } \\
& p_{c} \rightarrow * p_{a} \rightarrow=(1,1,1,1,1), p_{c} \rightarrow * p_{b} \rightarrow=p_{c} \rightarrow * p_{c} \rightarrow=p_{c} \rightarrow, p_{c} \rightarrow * p_{d} \rightarrow=(1,1,1,1,1), p_{c} \rightarrow * p_{1} \rightarrow=p_{b} \rightarrow \text {; } \\
& p_{d} \rightarrow * p_{a} \rightarrow=p_{d} \rightarrow, p_{d} \rightarrow * p_{b} \rightarrow=p_{d} \rightarrow * p_{c} \rightarrow=(1,1,1,1,1), p_{d} \rightarrow * p_{d} \rightarrow=p_{d} \rightarrow, p_{d} \rightarrow * p_{1} \rightarrow=p_{d} \rightarrow \text {. } \\
& \text { Denote } p_{a b} \rightarrow=p_{a} \rightarrow * p_{b} \rightarrow=(1,1,1,1,1) \text {, then } p_{a b} \rightarrow * p_{a} \rightarrow=p_{a b} \rightarrow * p_{b} \rightarrow=p_{a b} \rightarrow * p_{c} \rightarrow=p_{a b} \rightarrow * p_{d} \rightarrow
\end{aligned}
$$

Table 18. Pseudo BE-algebra and commutative neutrosophic triplet groups (1).

\begin{tabular}{llllll}
\hline$\rightarrow$ & $\boldsymbol{a}$ & $\boldsymbol{b}$ & $\boldsymbol{c}$ & $\boldsymbol{d}$ & $\mathbf{1}$ \\
\hline $\boldsymbol{a}$ & 1 & $c$ & $c$ & 1 & 1 \\
$\boldsymbol{b}$ & $d$ & 1 & 1 & $d$ & 1 \\
$c$ & $d$ & 1 & 1 & $d$ & 1 \\
$\boldsymbol{d}$ & 1 & $c$ & $c$ & 1 & 1 \\
$\mathbf{1}$ & $a$ & $b$ & $c$ & $d$ & 1 \\
\hline
\end{tabular}

Table 19. Pseudo BE-algebra and commutative neutrosophic triplet groups (2).

\begin{tabular}{llllll}
\hline$\rightsquigarrow$ & $\boldsymbol{a}$ & $\boldsymbol{b}$ & $\boldsymbol{c}$ & $\boldsymbol{d}$ & $\mathbf{1}$ \\
\hline $\boldsymbol{a}$ & 1 & $b$ & $c$ & 1 & 1 \\
$\boldsymbol{b}$ & $d$ & 1 & 1 & $d$ & 1 \\
$c$ & $d$ & 1 & 1 & $d$ & 1 \\
$\boldsymbol{d}$ & 1 & $b$ & $c$ & 1 & 1 \\
$\mathbf{1}$ & $a$ & $b$ & $c$ & $d$ & 1 \\
\hline
\end{tabular}

Table 20. Pseudo BE-algebra and commutative neutrosophic triplet groups (3).

\begin{tabular}{ccccc}
\hline$*$ & $p_{\boldsymbol{a}} \rightarrow$ & $p_{\boldsymbol{b}} \rightarrow$ & $p_{a b} \rightarrow$ & $p_{\mathbf{1}} \rightarrow$ \\
\hline$p_{\boldsymbol{a}} \rightarrow$ & $p_{a} \rightarrow$ & $p_{a b} \rightarrow$ & $p_{a b} \rightarrow$ & $p_{a} \rightarrow$ \\
$p_{\boldsymbol{b}} \rightarrow$ & $p_{a b} \rightarrow$ & $p_{b} \rightarrow$ & $p_{a b} \rightarrow$ & $p_{b} \rightarrow$ \\
$p_{a b} \rightarrow$ & $p_{a b} \rightarrow$ & $p_{a b} \rightarrow$ & $p_{a b} \rightarrow$ & $p_{a b} \rightarrow$ \\
$p_{\mathbf{1}} \rightarrow$ & $p_{a} \rightarrow$ & $p_{b} \rightarrow$ & $p_{a b} \rightarrow$ & $p_{1} \rightarrow$ \\
\hline
\end{tabular}

Similarly, we can verify the following:

$$
\begin{aligned}
& p_{a}{ }^{\rightsquigarrow}=(1, b, c, 1,1), p_{b}=(d, 1,1, d, 1), p_{c}^{\rightsquigarrow}=(d, 1,1, d, 1), p_{d}^{\rightsquigarrow}=(1, b, c, 1,1), p_{1}^{\rightsquigarrow}=(a, b, c, d, 1) .
\end{aligned}
$$

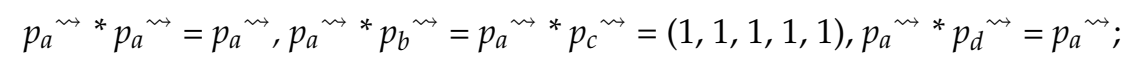

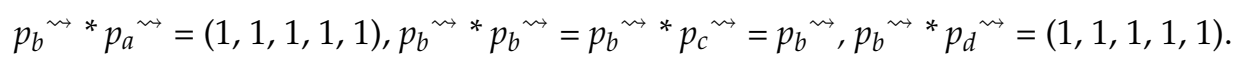

Denote $p_{a b}{ }^{\rightsquigarrow}=p_{a}{ }^{\rightsquigarrow *} p_{b} \rightsquigarrow=(1,1,1,1,1)$, then $M^{\rightsquigarrow}(X)=\left\{p_{a} \rightsquigarrow, p_{b}, p_{a b}{ }^{\rightsquigarrow}, p_{1}{ }^{\rightsquigarrow}\right\}$ and its Cayley table is Table 21. Obviously, $\left(M^{\sim}(X), *\right)$ is a commutative neutrosophic triplet group. 
Table 21. Pseudo BE-algebra and commutative neutrosophic triplet groups (4).

\begin{tabular}{|c|c|c|c|c|}
\hline * & $p_{a}^{\rightsquigarrow}$ & $p_{b}^{\rightsquigarrow}$ & $p_{a b} \rightsquigarrow$ & $p_{1} \rightsquigarrow$ \\
\hline$p_{a}^{\rightsquigarrow}$ & $p_{a}^{\rightsquigarrow}$ & $p_{a b} \rightsquigarrow$ & $p_{a b} \rightsquigarrow$ & $p_{a} \rightsquigarrow$ \\
\hline$p_{b}^{\rightsquigarrow}$ & $p_{a b} \rightsquigarrow$ & $p_{b}^{\rightsquigarrow}$ & $p_{a b} \rightsquigarrow$ & $p_{b}^{\rightsquigarrow}$ \\
\hline$p_{a b} \rightsquigarrow$ & $p_{a b} \rightsquigarrow$ & $p_{a b}^{\rightsquigarrow}$ & $p_{a b} \rightsquigarrow$ & $p_{a b} \rightsquigarrow$ \\
\hline$p_{1}^{\rightsquigarrow}$ & $p_{a} \rightsquigarrow$ & $p_{b}^{\rightsquigarrow}$ & $p_{a b^{n}}^{\rightsquigarrow}$ & $p_{1} \rightsquigarrow$ \\
\hline
\end{tabular}

Now, we consider $M(X)$. Since the following:

$$
\begin{aligned}
& p_{b} \rightarrow=p_{c} \rightarrow=(d, 1,1, d, 1)=p_{b}=p_{c} \rightsquigarrow, p_{a} \rightarrow=p_{d} \rightarrow=(1, c, c, 1,1), p_{a}=p_{d}^{\rightsquigarrow}=(1, b, c, 1,1) ; \\
& p_{a} \rightarrow * p_{a}{ }^{\rightsquigarrow}=p_{a} \rightarrow, p_{a} \rightsquigarrow * p_{a} \rightarrow=p_{a} \rightarrow ; p_{a} \rightarrow * p_{b} \rightsquigarrow(1,1,1,1,1)=p_{a b} \rightarrow=p_{a b} \rightsquigarrow, p_{b}{ }^{*} p_{a} \rightarrow=(1,1,1,1,1) .
\end{aligned}
$$

Hence, $M(X)=\left\{p_{a} \rightarrow, p_{a} \rightsquigarrow, p_{b} \rightarrow, p_{a b} \rightarrow, p_{1} \rightarrow\right\}$, and Table 22 is its Cayley table (it is a commutative neutrosophic triplet group).

\begin{tabular}{|c|c|c|c|c|c|}
\hline * & $p_{a} \rightarrow$ & $p_{a}^{\rightsquigarrow}$ & $p_{b} \rightarrow$ & $p_{a b} \rightarrow$ & $p_{1} \rightarrow$ \\
\hline$p_{a} \rightarrow$ & $p_{a} \rightarrow$ & $p_{a} \rightarrow$ & $p_{a b} \rightarrow$ & $p_{a b} \rightarrow$ & $p_{a} \rightarrow$ \\
\hline$p_{a}^{\rightsquigarrow}$ & $p_{a} \rightarrow$ & $p_{a}^{\rightsquigarrow}$ & $p_{a b} \rightarrow$ & $p_{a b} \rightarrow$ & $p_{a}^{\rightsquigarrow}$ \\
\hline$p_{b} \rightarrow$ & $p_{a b} \rightarrow$ & $p_{a b} \rightarrow$ & $p_{b} \rightarrow$ & $p_{a b} \rightarrow$ & $p_{b} \rightarrow$ \\
\hline$p_{a b} \rightarrow$ & $p_{a b} \rightarrow$ & $p_{a b} \rightarrow$ & $p_{a b} \rightarrow$ & $p_{a b} \rightarrow$ & $p_{a b} \rightarrow$ \\
\hline$p_{1} \rightarrow$ & & $p_{a} \rightsquigarrow$ & & $p_{a b} \rightarrow$ & $p_{1} \rightarrow$ \\
\hline
\end{tabular}

Table 22. Pseudo BE-algebra and commutative neutrosophic triplet groups (5).

Remark 3. Through the discussions of Examples 10 and 11 above, we get the following important revelations: (1) $\left(M^{\rightarrow}(X), *\right),\left(M^{\rightsquigarrow}(X), *\right)$, and $\left(M(X),{ }^{*}\right)$ are usually three different semi-groups; $(2)\left(M^{\rightarrow}(X),{ }^{*}\right)$ and $\left(M^{\rightsquigarrow}(X)\right.$, *) are all sub-semi-groups of $\left(M(X),{ }^{*}\right)$, which can also be proved from their definitions; $(3)(M \rightarrow(X), *),\left(M^{\rightsquigarrow}(X)\right.$, $\left.{ }^{*}\right)$, and $\left(M(X),{ }^{*}\right)$ may be neutrosophic triplet groups. Under what circumstances they will become neutrosophic triplet groups, will be examined in the next study.

\section{Conclusions}

In this paper, the concepts of neutrosophic triplet loops (groups) are further generalized, and some new concepts of generalized neutrosophic triplet structures are proposed, including (l-l)-type, $(l-r)$-type, $(r-l)$-type, $(r$-r $r$-type, $(l-l r)$-type, $(r$-lr)-type, $(l r-l)$-type, and $(l r-r)$-type quasi neutrosophic triplet loops (groups), and their basic properties are discussed. In particular, as a corollary of these new properties, an important result is proved. For any commutative neutrosophic triplet group, its every element has only one neutral element. At the same time, the BE-algebras and its various extensions (including CI-algebras, pseudo BE-algebras, and pseudo CI-algebras) have been studied, and some related generalized neutrosophic triplet structures that are contained in these algebras are presented. Moreover, the concept of adjoint semigroups of (generalized) BE-algebras are proposed for the first time, abundant examples are given, and some new results are obtained.

Author Contributions: X.Z. and X.W. initiated the research and wrote the paper; F.S. supervised the research work and provided helpful suggestions; and M.H. participated in some of the research work.

Funding: This research received no external funding.

Acknowledgments: This work was supported by the National Natural Science Foundation of China (Grant Nos. 61573240, 61473239).

Conflicts of Interest: The authors declare no conflict of interest. 


\section{References}

1. Molaei, M.R. Generalized groups. Bull. Inst. Polit. Di. Iase Fasc. 1999, 3, 21-24.

2. Molaei, M.R. Generalized actions. In Proceedings of the First International Conference on Geometry, Integrability and Quantization, Varna, Bulgaria, 1-10 September 1999; pp. 175-180.

3. Araujo, J.; Konieczny, J. Molaei's Generalized groups are completely simple semigroups. Bull. Polytech. Inst. Iassy 2002, 48, 1-5.

4. Adeniran, J.O.; Akinmoyewa, J.T.; Solarin, A.R.T.; Jaiyeola, T.G. On some algebraic properties of generalized groups. Acta Math. Acad. 2011, 27, 23-30.

5. Smarandache, F. Neutrosophic Perspectives: Triplets, Duplets, Multisets, Hybrid Operators, Modal Logic, Hedge Algebras. and Applications; Pons Publishing House: Brussels, Belgium, 2017.

6. Smarandache, F.; Ali, M. Neutrosophic triplet group. Neural Comput. Appl. 2018, 29, 595-601. [CrossRef]

7. Smarandache, F. Neutrosophic set-A generialization of the intuituionistics fuzzy sets. Int. J. Pure Appl. Math. 2005, 3, 287-297.

8. Liu, P.D.; Shi, L.L. Some Neutrosophic uncertain linguistic number Heronian mean operators and their application to multi-attribute group decision making. Neural Comput. Appl. 2017, 28, 1079-1093. [CrossRef]

9. Ye, J.; Du, S. Some distances, similarity and entropy measures for interval-valued neutrosophic sets and their relationship. Int. J. Mach. Learn Cybern. 2018. [CrossRef]

10. Zhang, X.H.; Ma, Y.C.; Smarandache, F. Neutrosophic regular filters and fuzzy regular filters in pseudo-BCI algebras. Neutrosophic Sets Syst. 2017, 17, 10-15.

11. Zhang, X.H.; Bo, C.X.; Smarandache, F.; Dai, J.H. New inclusion relation of neutrosophic sets with applications and related lattice structure. Int. J. Mach. Learn. Cyber. 2018. [CrossRef]

12. Zhang, X.H.; Smarandache, F.; Liang, X.L. Neutrosophic duplet semi-group and cancellable neutrosophic triplet groups. Symmetry 2017, 9, 275. [CrossRef]

13. Jaiyeola, T.G.; Smarandache, F. Inverse properties in neutrosophic triplet loop and their application to cryptography. Algorithms 2018, 11, 32. [CrossRef]

14. Bal, M.; Shalla, M.M.; Olgun, N. Neutrosophic triplet cosets and quotient groups. Symmetry 2018, 10, 126. [CrossRef]

15. Zhang, X.H.; Smarandache, F.; Ali, M.; Liang, X.L. Commutative neutrosophic triplet group and neutrohomomorphism basic theorem. Ital. J. Pure Appl. Math. 2018, in press.

16. Fleischer, I. Every BCK-algebra is a set of residuables in an integral pomonoid. J. Algebra 1980, 119, 360-365. [CrossRef]

17. Huang, W. On BCI-algebras and semigroups. Math. Jpn. 1995, 42, 59-64.

18. Zhang, X.H.; Ye, R.F. BZ-algebra and group. J. Math. Phys. Sci. 1995, 29, 223-233.

19. Dudek, W.A.; Zhang, X.H. On atoms in BCC-algebras. Discuss. Math. Algebra Stoch. Methods 1995, 15, 81-85.

20. Huang, W.; Liu, F. On the adjoint semigroups of p-separable BCI-algebras. Semigroup Forum 1999, 58, 317-322. [CrossRef]

21. Zhang, X.H.; Wang, Y.Q.; Dudek, W.A. T-ideals in BZ-algebras and T-type BZ-algebras. Indian J. Pure Appl. Math. 2003, 34, 1559-1570.

22. Zhang, X.H. Fuzzy Logics and Algebraic Analysis; Science Press: Beijing, China, 2008.

23. Zhang, X.H.; Dudek, W.A. BIK+-logic and non-commutative fuzzy logics. Fuzzy Syst. Math. 2009, 23, 8-20.

24. Zhang, X.H.; Jun, Y.B. Anti-grouped pseudo-BCI algebras and anti-grouped pseudo-BCI filters. Fuzzy Syst. Math. 2014, 28, 21-33.

25. Zhang, X.H. Fuzzy anti-grouped filters and fuzzy normal filters in pseudo-BCI algebras. J. Intell. Fuzzy Syst. 2017, 33, 1767-1774. [CrossRef]

26. Zhang, X.H.; Park, C.; Wu, S.P. Soft set theoretical approach to pseudo-BCI algebras. J. Intell. Fuzzy Syst. 2018, 34, 559-568. [CrossRef]

27. Kim, H.S.; Kim, Y.H. On BE-algebras. Sci. Math. Jpn. 2007, 66, 113-116.

28. Ahn, S.S.; So, Y.H. On ideals and upper sets in BE-algebras. Sci. Math. Jpn. 2008, 68, 279-285.

29. Walendziak, A. On commutative BE-algebras. Sci. Math. Jpn. 2009, 69, 281-284.

30. Meng, B.L. On filters in BE-algebras. Sci. Math. Jpn. 2010, 71, 201-207.

31. Walendziak, A. On normal filters and congruence relations in BE-algebras. Comment. Math. 2012, 52, 199-205.

32. Sambasiva Rao, M. A Course in BE-Algebras; Springer: Berlin, Germany, 2018. 
33. Borzooei, R.A.; Saeid, A.B.; Rezaei, A.; Radfar, A.; Ameri, R. On pseudo BE-algebras. Discuss. Math. Gen. Algebra Appl. 2013, 33, 95-108. [CrossRef]

34. Ciungu, L.C. Commutative pseudo BE-algebras. Iran. J. Fuzzy Syst. 2016, 13, 131-144.

35. Meng, B.L. CI-algebras. Sci. Math. Jpn. 2010, 71, 11-17.

36. Meng, B.L. Atoms in CI-algebras and singular CI-algebras. Sci. Math. Jpn. 2010, 72, 67-72.

37. Meng, B.L. Closed filters in CI-algebras. Sci. Math. Jpn. 2010, 71, 265-270.

38. Kim, K.H. A note on CI-algebras. Int. Math. Forum 2011, 6, 1-5.

39. Jun, Y.B.; Lee, K.J.; Roh, E.H. Ideals and filters in CI-algebras based on bipolar-valued fuzzy sets. Ann. Fuzz. Math. Inf. 2012, 4, 109-121.

40. Sabhapandit, P.; Pathak, K. On homomorphisms in CI-algebras. Int. J. Math. Arch. 2018, 9, 33-36.

41. Neggers, J.; Ahn, S.S.; Kim, H.S. On Q-algebras. Int. J. Math. Math. Sci. 2001, 27, 749-757. [CrossRef]

42. Saeid, A.B. CI-algebra is equivalent to dual Q-algebra. J. Egypt. Math. Soc. 2013, 21, 1-2. [CrossRef]

43. Walendziak, A. Pseudo-BCH-algebras. Discuss. Math. Gen. Algebra Appl. 2015, 35, 5-19. [CrossRef]

44. Jun, Y.B.; Kim, H.S.; Ahn, S.S. Structures of pseudo ideal and pseudo atom in a pseudo Q-algebra. Kyungpook Math. J. 2016, 56, 95-106. [CrossRef]

45. Rezaei, A.; Saeid, A.B.; Walendziak, A. Some results on pseudo-Q algebras. Ann. Univ. Paedagog. Crac. Stud. Math. 2017, 16, 61-72. [CrossRef]

46. Bajalan, S.A.; Ozbal, S.A. Some properties and homomorphisms of pseudo-Q algebras. J. Cont. Appl. Math. 2016, 6, 3-17.

(C) 2018 by the authors. Licensee MDPI, Basel, Switzerland. This article is an open access article distributed under the terms and conditions of the Creative Commons Attribution (CC BY) license (http:/ / creativecommons.org/licenses/by/4.0/). 